\title{
ON ORDERINGS INDUCED BY THE LOEWNER PARTIAL ORDERING
}

Abstract. The partial ordering induced by the Loewner partial ordering on the convex cone comprising all matrices which multiplied by a given positive definite matrix become nonnegative definite is considered. Its relation to orderings which are induced by the Loewner partial ordering of the squares of matrices is presented. Some extensions of the latter orderings and their comparison to star orderings are given.

1. Introduction and preliminaries. Let $M_{m, n}, M_{n}, M_{n}^{>}$, and $M_{n}^{>}$ stand for the set of $m \times n$ complex matrices, the set $M_{n, n}$, the subset of $M_{n}$ consisting of Hermitian nonnegative definite matrices, and the subset of $M_{\bar{n}}^{\geq}$consisting of positive definite matrices, respectively. Given $\mathbf{A} \in$ $M_{m, n}$ the symbols $\mathbf{A}^{*}, R(\mathbf{A}), \operatorname{tr}(\mathbf{A}), \varrho(\mathbf{A}),\|\mathbf{A}\|$, and $r(\mathbf{A})$ will denote the conjugate transpose, range, trace, spectral radius, spectral norm, and rank of $\mathbf{A}$, respectively. Further, for $\mathbf{N} \in M_{n}^{>}$let $\|\mathbf{A}\|_{\mathbf{N}}=\left\|\mathbf{N}^{1 / 2} \mathbf{A N}^{-1 / 2}\right\|$. The symbols $\mathbf{P}_{\mathbf{A}}$ and $\mathbf{Q}_{\mathbf{A}}$ will stand for the orthogonal projectors onto the orthocomplements of $R(\mathbf{A})$ and $R\left(\mathbf{A}^{*}\right)$ under the standard inner product, respectively. For $\mathbf{M} \in M_{m}^{>}$and $\mathbf{N} \in M_{n}^{>}$consider the following conditions:

$$
\begin{aligned}
\mathbf{A G A} & =\mathbf{A}, \\
\mathbf{G A G} & =\mathbf{G}, \\
(\mathbf{A G})^{*} \mathbf{M} & =\mathbf{M A G}, \\
(\mathbf{G A})^{*} \mathbf{N} & =\mathbf{N G A},
\end{aligned}
$$

where $\mathbf{G} \in M_{n, m}$. The following terminology and notation is used for every $\mathbf{G}$ which satisfies the specified set of conditions:

(1.1): a generalized inverse ( $g$-inverse) of $\mathbf{A}$, denoted by $\mathbf{A}^{-}$;

(1.1) and (1.3): an $\mathbf{M}$-least squares g-inverse of $\mathbf{A}$, denoted by $\mathbf{A}_{l(\mathbf{M})}^{-}$;

1991 Mathematics Subject Classification: 15A09, 15A60.

Key words and phrases: Loewner ordering, star ordering, matrix norm, spectral radius. 
(1.1) and (1.4): a minimum $\mathbf{N}$-norm g-inverse of $\mathbf{A}$, denoted by $\mathbf{A}_{m(\mathbf{N})}^{-}$;

(1.1), (1.2), and (1.3): an $\mathbf{M}$-least squares reflexive $g$-inverse of $\mathbf{A}$, denoted by $\mathbf{A}_{l(\mathbf{M}) r}^{-}$;

(1.1), (1.2), and (1.4): a minimum $\mathbf{N}$-norm reflexive $g$-inverse of $\mathbf{A}$, denoted by $\mathbf{A}_{m(\mathbf{N}) r}^{-}$;

(1.1), (1.2), (1.3), and (1.4): a minimum $\mathbf{N}$-norm $\mathbf{M}$-least squares $g$ inverse of $\mathbf{A}$ (it is unique), denoted by $\mathbf{A}_{\mathbf{M N}}^{+}$.

The sets of all $g$-inverses in each case will be denoted by $\left\{\mathbf{A}_{l(\mathbf{M})}^{-}\right\}$etc. When $\mathbf{M}$ or $\mathbf{N}$ are identity matrices, we drop the subscripts.

For $\mathbf{A}, \mathbf{B} \in M_{n}^{\geq}$we say that $\mathbf{A}$ is below $\mathbf{B}$ with respect to the Loewner partial ordering, and we write $\mathbf{A} \leq_{\mathrm{L}} \mathbf{B}$, if $\mathbf{B}-\mathbf{A} \in M_{n}^{\geq}$. This ordering can be characterized in different ways. One of them, which follows [1], is presented in Lemma 1.

Lemma 1. For any $\mathbf{A}, \mathbf{B} \in M_{\bar{n}}^{\geq}$the following statements are equivalent:

(a) $\mathbf{A} \leq_{L} \mathbf{B}$

(b) $\varrho\left(\mathbf{B}^{+} \mathbf{A}\right) \leq 1$ and $R(\mathbf{A}) \subseteq R(\mathbf{B})$,

(c) $\varrho\left(\mathbf{B}^{-} \mathbf{A}\right) \leq 1$ and $R(\mathbf{A}) \subseteq R(\mathbf{B})$, the first condition being independent of the choice of $\mathbf{B}^{-}$.

As is well known (see e.g. Horn and Johnson [7, p. 470]) a partial ordering on the set of matrices can be defined by specifying a convex cone and declaring that $\mathbf{A}$ is below $\mathbf{B}$ if $\mathbf{B}-\mathbf{A}$ lies in the cone. In the present paper we consider the convex cone $M_{n}^{\geq}(\mathbf{S})$, with fixed $\mathbf{S} \in M_{n}^{>}$, defined as follows:

$$
M_{n}^{\geq}(\mathbf{S})=\left\{\mathbf{A}: \mathbf{A} \in M_{n}, \mathbf{S A} \in M_{n}^{\geq}\right\} .
$$

The matrices in this cone have the following properties. They represent selfadjoint and nonnegative definite transformations with respect to the scalar product defined by $\mathbf{S}$ (see e.g. [5, p. 311]). In statistical applications such matrices appear in the problem of estimation in the linear model (see e.g. [1], [9]). In the model with the dispersion matrix of the vector of observations proportional to a positive definite matrix $\mathbf{V}$ the linear transformations from the cone $M_{n}^{\geq}\left(\mathbf{V}^{-1}\right)$ form the complete class of linear estimators of the vector of expectations. The dispersion matrices of the estimators can be compared via the ordering described in Section 3.

In the second section we characterize the partial ordering on $M_{n}^{\geq}(\mathbf{S})$ in a similar way to Lemma 1.

Stępniak [11] considered the equivalence of the Loewner partial ordering of nonnegative definite matrices to the Loewner partial ordering of their squares. In Section 3 we define and characterize new orderings, similar to the latter, and we compare these orderings with the ones from Section 2. The main result of the paper is given in the Theorem stated there. 
Several orderings in $M_{m, n}$ are considered in the literature. In Section 4 we are interested in the star ordering introduced in [6], especially, in the results of Mitra [8] and Baksalary and Mitra [2]. We compare their results with extensions of the results presented in Section 3.

2. Orderings induced by the Loewner partial order. The partial ordering on the convex cone $M_{n}^{\geq}(\mathbf{S})$ is specified in the following

Definition 1. iFor $\mathbf{A}, \mathbf{B} \in M_{n}^{\geq}(\mathbf{S})$ we say that $\mathbf{A}$ is below $\mathbf{B}$ with respect to the $\mathbf{S L}$-partial ordering, and we write $\mathbf{A} \leq \mathbf{S L}_{\mathbf{S}} \mathbf{B}$, if $\mathbf{B}-\mathbf{A} \in M_{n}^{\geq}(\mathbf{S})$.

Note $1 . \mathbf{A} \leq_{\mathbf{S L}} \mathbf{B}$ if and only if $\mathbf{S A} \leq_{\mathrm{L}} \mathbf{S B}$.

The next lemma is useful for proving the results of the paper.

Lemma 2. For any $\mathbf{A}, \mathbf{B} \in M_{m, n}$ the following statements are equivalent:

(a) $\varrho\left(\mathbf{B}^{-} \mathbf{A}\right)=\varrho\left(\mathbf{B}^{+} \mathbf{A}\right)$ for every $\mathbf{B}^{-}$,

(b) there exists $d>0$ such that $\varrho\left(\mathbf{B}^{-} \mathbf{A}\right) \leq d$ for every $\mathbf{B}^{-}$,

(c) $R(\mathbf{A}) \subseteq R(\mathbf{B})$ and $R\left(\mathbf{A}^{*}\right) \subseteq R\left(\mathbf{B}^{*}\right)$.

Proof. The implication $(a) \Rightarrow(b)$ is obvious. The general formula for $\mathbf{B}^{-}$is

$$
\mathbf{B}^{-}=\mathbf{B}^{+}+\mathbf{W P}_{\mathbf{B}}+\mathbf{Q}_{\mathbf{B}} \mathbf{U},
$$

with $\mathbf{W}$ and $\mathbf{U}$ arbitrary in $M_{n, m}$ (cf. [10, p. 20]). Observing that for $\mathbf{C}, \mathbf{D} \in M_{n}$,

$$
\varrho(\mathbf{C}+\mathbf{D}) \geq \frac{1}{n}|\operatorname{tr}(\mathbf{C}+\mathbf{D})| \geq \frac{1}{n}|| \operatorname{tr}(\mathbf{C})|-| \operatorname{tr}(\mathbf{D})||,
$$

and putting in (2.1), $\mathbf{W}=\alpha \mathbf{A}^{*}$ and $\mathbf{U}=\mathbf{0}$, and next $\mathbf{W}=\mathbf{0}$ and $\mathbf{U}=\alpha \mathbf{A}^{*}$, it follows that (b) implies (c). Because of the form (2.1) of $\mathbf{B}^{-}$, and since $\varrho\left(\mathbf{B}^{-} \mathbf{A}\right)=\varrho\left(\mathbf{A} \mathbf{B}^{-}\right)$, it is clear that (c) implies (a).

Similarly to [1] we characterize this new ordering in the following

Proposition 1. Let $\mathbf{M}, \mathbf{N}$, and $\mathbf{S} \in M_{n}^{>}$be fixed. For any $\mathbf{A}, \mathbf{B} \in$ $M_{n}^{\geq}(\mathbf{S})$ the following statements are equivalent:
(a) $\mathbf{A} \leq \mathbf{S L} \mathbf{B}$
(b) $\varrho\left(\mathbf{B}^{-} \mathbf{A}\right) \leq 1$ for every $\mathbf{B}^{-}$,
(c) $\varrho\left(\mathbf{B}_{l(\mathbf{M})}^{-} \mathbf{A}\right) \leq 1$ for every $\mathbf{B}_{l(\mathbf{M})}^{-}$,
(d) $\varrho\left(\mathbf{B}_{m(\mathbf{N})}^{-} \mathbf{A}\right) \leq 1$ for every $\mathbf{B}_{m(\mathbf{N})}^{-}$,
(e) $\varrho\left(\mathbf{B}^{-} \mathbf{A}\right) \leq 1$ for some $\mathbf{B}^{-}$and $R(\mathbf{A}) \subseteq R(\mathbf{B})$
(f) $\varrho\left(\mathbf{B}_{\mathbf{M N}}^{+} \mathbf{A}\right) \leq 1$ and $R(\mathbf{A}) \subseteq R(\mathbf{B})$.

Proof. By Definition 1, Note 1, Lemma 1, and the observation that $\mathbf{B}_{\mathbf{M N}}^{+} \mathbf{S}^{-1}$ is a generalized inverse of $\mathbf{S B}$, the following statements are equivalent: 
- $\mathbf{A} \leq_{\mathrm{SL}} \mathbf{B}$,

- $\varrho\left[\left(\mathbf{B}_{\mathbf{M N}}^{+} \mathbf{S}^{-1}\right) \mathbf{S A}\right] \leq 1$ and $R(\mathbf{S A}) \subseteq R(\mathbf{S B})$,

- $\varrho\left(\mathbf{B}_{\mathbf{M N}}^{+} \mathbf{A}\right) \leq 1$ and $R(\mathbf{A}) \subseteq R(\mathbf{B})$.

Further, it is clear that for $\mathbf{A}, \mathbf{B} \in M_{n}^{\geq}(\mathbf{S}), R(\mathbf{A}) \subseteq R(\mathbf{B})$ if and only if $R\left(\mathbf{A}^{*}\right) \subseteq R\left(\mathbf{B}^{*}\right)$ and hence, the equivalence of (b), (e) and (f) follows from Lemma 2.

Now assume that (d) holds. Then, using the following general representation of $\mathbf{B}_{m(\mathbf{N})}^{-}[10$, p. 45]:

$$
\mathbf{B}_{m(\mathbf{N})}^{-}=\mathbf{N}^{-1} \mathbf{B}^{*}\left(\mathbf{B} \mathbf{N}^{-1} \mathbf{B}^{*}\right)^{+}+\mathbf{U} \mathbf{P}_{\mathbf{B}}
$$

with arbitrary $\mathbf{U} \in M_{n}$, and putting $\mathbf{U}=\alpha \mathbf{A}^{*}$, where $\alpha$ is an arbitrary real number, we observe that, by $(2.2)$, the inequalities

$$
1 \geq \varrho\left(\mathbf{B}_{m(\mathbf{N})}^{-} \mathbf{A}\right) \geq \frac{1}{n}|| \operatorname{tr}\left[\mathbf{N}^{-1} \mathbf{B}^{*}\left(\mathbf{B N}^{-1} \mathbf{B}\right)^{+} \mathbf{A}\right]|-| \alpha \operatorname{tr}\left(\mathbf{A}^{*} \mathbf{P}_{\mathbf{B}} \mathbf{A}\right)||
$$

cannot hold for all real numbers $\alpha$ unless $\mathbf{P}_{\mathbf{B}} \mathbf{A}=\mathbf{0}$.

Assuming (c) and using the general representation of $\mathbf{B}_{l(\mathbf{M})}^{-}[10$, p. 49]:

$$
\mathbf{B}_{l(\mathbf{M})}^{-}=\left(\mathbf{B}^{*} \mathbf{M B}\right)^{+} \mathbf{B}^{*} \mathbf{M}+\mathbf{Q}_{\mathbf{B}} \mathbf{W},
$$

with arbitrary $\mathbf{W} \in M_{n}$, we obtain $\mathbf{Q}_{\mathbf{B}} \mathbf{A}^{*}=\mathbf{0}$. Thus, the implications $(\mathrm{c}) \Rightarrow(\mathrm{e})$ and $(\mathrm{d}) \Rightarrow(\mathrm{e})$ hold; since $(\mathrm{b}) \Rightarrow(\mathrm{c})$ and $(\mathrm{b}) \Rightarrow(\mathrm{d})$ are obvious, the proof is complete.

Note that the characterization given in Proposition 1 for matrices from $M_{n}^{\geq}(\mathbf{S})$ does not depend on the matrix $\mathbf{S}$, and that conditions (c) and (d) involve the narrower classes of $g$-inverses of $\mathbf{B}$.

Theorem 3 of Bekker [4] gives equivalent conditions for two nonnegative definite matrices to be Loewner ordered. We present a counterpart of Bekker's result for matrices from $M_{n}^{\geq}(\mathbf{S})$. This result is used in the next section.

Note 2. For any $\mathbf{A}, \mathbf{B} \in M_{n}^{\geq}(\mathbf{S})$,

$\mathbf{A} \leq_{\mathbf{S L}} \mathbf{B}$ if and only if $R(\mathbf{A}) \subseteq R(\mathbf{B})$ and $\mathbf{A B}^{-} \mathbf{A} \leq_{\mathbf{S L}} \mathbf{A}$ for some $\mathbf{B}^{-}$.

P r o of. The equivalence follows from Proposition 1 observing that under the condition $R(\mathbf{A}) \subseteq R(\mathbf{B})$,

$$
\mathbf{A B}^{-} \mathbf{A}=\mathbf{A}(\mathbf{S B})^{-} \mathbf{S A}=\mathbf{A}(\mathbf{S B})^{+} \mathbf{S A} \in M_{n}^{\geq}(\mathbf{S}) \text {. }
$$

3. Orderings induced by the Loewner partial ordering of the squares of matrices. Extending results given in [11], we consider a family of partial orderings defined on the cone $M_{n}^{\geq}(\mathbf{S})$. 
Definition 2. For $\mathbf{A}, \mathbf{B} \in M_{n}^{\geq}(\mathbf{S})$ and a given matrix $\mathbf{M} \in M_{n}^{>}$we say that $\mathbf{A}$ is below $\mathbf{B}$ with respect to the left star $\mathbf{M L}$-partial ordering, and we write $\mathbf{A} * \leq_{\mathbf{M L}} \mathbf{B}$, if $\mathbf{A}^{*} \mathbf{M A} \leq_{\mathrm{L}} \mathbf{B}^{*} \mathbf{M B}$; for a given matrix $\mathbf{N} \in M_{n}^{>}$we say that $\mathbf{A}$ is below $\mathbf{B}$ with respect to the right star $\mathbf{N L}$-partial ordering, and we write $\mathbf{A} \leq *_{\mathbf{N L}} \mathbf{B}$, if $\mathbf{A} \mathbf{N}^{-1} \mathbf{A}^{*} \leq_{\mathrm{L}} \mathbf{B} \mathbf{N}^{-1} \mathbf{B}^{*}$.

For $\mathbf{A}, \mathbf{B} \in M_{n}^{\geq}$Stȩpniak [11] considered the equivalence of the orderings $\mathbf{A} \leq_{\mathrm{L}} \mathbf{B}$ and $\mathbf{A} * \leq_{\mathbf{I L}} \mathbf{B}$ (or $\mathbf{A} \leq *_{\mathbf{I L}} \mathbf{B}$ ) and gave some necessary and sufficient conditions for it. In this paper we consider the equivalence of the ordering $\mathbf{A} \leq_{\mathbf{S L}} \mathbf{B}$ and the family of orderings specified in Definition 2.

The following propositions providing some characterizations of these orderings are counterparts of Proposition 1.

Proposition 2. Let $\mathbf{M}, \mathbf{N}$, and $\mathbf{S} \in M_{n}^{>}$be fixed. For any $\mathbf{A}, \mathbf{B} \in$ $M_{n}^{\geq}(\mathbf{S})$ the following statements are equivalent:

(a) $\mathbf{A} * \leq_{\mathrm{ML}} \mathbf{B}$,

(b) $\left\|\mathbf{A B}_{l(\mathbf{M})}^{-}\right\|_{\mathbf{M}} \leq 1$ for every $\mathbf{B}_{l(\mathbf{M})}^{-}$,

(c) $\left\|\mathbf{A B}_{l(\mathbf{M}) r}^{-}\right\|_{\mathbf{M}} \leq 1$ for every $\mathbf{B}_{l(\mathbf{M}) r}^{-}$, and if $\mathbf{B}=\mathbf{0}$ then $\mathbf{A}=\mathbf{0}$,

(d) $\left\|\mathbf{A B}_{l(\mathbf{M})}^{-}\right\|_{\mathbf{M}} \leq 1$ for some $\mathbf{B}_{l(\mathbf{M})}^{-}$and $R\left(\mathbf{A}^{*}\right) \subseteq R\left(\mathbf{B}^{*}\right)$,

(e) $\left\|\mathbf{A B}_{\mathbf{M N}}^{+}\right\|_{\mathbf{M}} \leq 1$ and $R\left(\mathbf{A}^{*}\right) \subseteq R\left(\mathbf{B}^{*}\right)$.

Proof. By Definition 2 and Lemma $1, \mathbf{A} * \leq_{\mathbf{M L}} \mathbf{B}$ if and only if

$$
\varrho\left[\left(\mathbf{B}^{*} \mathbf{M B}\right)^{+} \mathbf{A}^{*} \mathbf{M} \mathbf{A}\right] \leq 1 \text { and } R\left(\mathbf{A}^{*}\right) \subseteq R\left(\mathbf{B}^{*}\right) .
$$

Since $\mathbf{B}_{\mathbf{M I}}^{+}=\left(\mathbf{B}^{*} \mathbf{M B}\right)^{+} \mathbf{B}^{*} \mathbf{M}$, it follows that

$$
\varrho\left[\left(\mathbf{B}^{*} \mathbf{M B}\right)^{+} \mathbf{A}^{*} \mathbf{M} \mathbf{A}\right]=\left\|\mathbf{A B}_{\mathbf{M I}}^{+}\right\|_{\mathbf{M}}^{2}
$$

and (3.1) is equivalent to

$$
\left\|\mathbf{A B}_{\mathbf{M I}}^{+}\right\|_{\mathbf{M}} \leq 1 \quad \text { and } \quad R\left(\mathbf{A}^{*}\right) \subseteq R\left(\mathbf{B}^{*}\right) .
$$

But, under the assumption $R\left(\mathbf{A}^{*}\right) \subseteq R\left(\mathbf{B}^{*}\right)$ and using the general formula (2.4) for $\mathbf{B}_{l(\mathbf{M})}^{-}$, we have

$$
\mathbf{A B}_{l(\mathbf{M})}^{-}=\mathbf{A B}_{\mathbf{M I}}^{+} \quad \text { for every } \mathbf{B}_{l(\mathbf{M})}^{-} .
$$

Combination of this equation with (3.2) yields $(\mathrm{a}) \Leftrightarrow(\mathrm{d}) \Leftrightarrow(\mathrm{e})$ and $(\mathrm{a}) \Rightarrow(\mathrm{b})$ $\Rightarrow(\mathrm{c})$.

Now assume that (c) holds. Then applying the following general form of $\mathbf{B}_{l(\mathbf{M}) r}^{-}$presented in $[10$, p. 49$]$ :

$$
\mathbf{B}_{l(\mathbf{M}) r}^{-}=\left(\mathbf{B}^{*} \mathbf{M B}\right)^{+} \mathbf{B}^{*} \mathbf{M}+\mathbf{Q}_{\mathbf{B}} \mathbf{W} \mathbf{B}^{*} \mathbf{M},
$$

with arbitrary $\mathbf{W} \in M_{n}$, and the inequalities

$$
1 \geq\left\|\mathbf{A B}_{\mathbf{M I}}^{+}+\mathbf{A Q}_{\mathbf{B}} \mathbf{W B}^{*} \mathbf{M}\right\|_{\mathbf{M}} \geq\left|\left\|\mathbf{A B}_{\mathbf{M I}}^{+}\right\|_{\mathbf{M}}-\left\|\mathbf{A Q}_{\mathbf{B}} \mathbf{W B}^{*} \mathbf{M}\right\|_{\mathbf{M}}\right|,
$$


for every $\mathbf{W} \in M_{n}$, it follows that $\mathbf{B}=\mathbf{0}$ or $\mathbf{A Q}_{\mathbf{B}}=\mathbf{0}$, which shows that $(\mathrm{c}) \Rightarrow(\mathrm{d})$.

Proposition 3. Let $\mathbf{M}, \mathbf{N}$, and $\mathbf{S} \in M_{n}^{>}$be fixed. For any $\mathbf{A}, \mathbf{B} \in$ $M_{n}^{\geq}(\mathbf{S})$ the following statements are equivalent:

(a) $\mathbf{A} \leq *_{N L} \mathbf{B}$

(b) $\left\|\mathbf{B}_{m(\mathbf{N})}^{-} \mathbf{A}\right\|_{\mathbf{N}} \leq 1$ for every $\mathbf{B}_{m(\mathbf{N})}^{-}$,

(c) $\left\|\mathbf{B}_{m(\mathbf{N}) r}^{-} \mathbf{A}\right\|_{\mathbf{N}} \leq 1$ for every $\mathbf{B}_{m(\mathbf{N}) r}^{-}$, and if $\mathbf{B}=\mathbf{0}$ then $\mathbf{A}=\mathbf{0}$,

(d) $\left\|\mathbf{B}_{m(\mathbf{N})}^{-} \mathbf{A}\right\|_{\mathbf{N}} \leq 1$ for some $\mathbf{B}_{m(\mathbf{N})}^{-}$and $R(\mathbf{A}) \subseteq R(\mathbf{B})$,

(e) $\left\|\mathbf{B}_{\mathbf{M N}}^{+} \mathbf{A}\right\|_{\mathbf{N}} \leq 1$ and $R(\mathbf{A}) \subseteq R(\mathbf{B})$.

Proof. The proof follows, similarly to Proposition 2, by observing that

$$
\mathbf{B}_{\mathbf{I N}}^{+}=\mathbf{N}^{-1} \mathbf{B}^{*}\left(\mathbf{B N}^{-1} \mathbf{B}^{*}\right)^{+},
$$

and using the general representation of $\mathbf{B}_{m(\mathbf{N}) r}^{-}[10$, p. 46]:

$$
\mathbf{B}_{m(\mathbf{N}) r}^{-}=\mathbf{N}^{-1} \mathbf{B}^{*}\left(\mathbf{B N}^{-1} \mathbf{B}^{*}\right)^{+}+\mathbf{N}^{-1} \mathbf{B}^{*} \mathbf{U} \mathbf{P}_{\mathbf{B}},
$$

with arbitrary $\mathbf{U} \in M_{n}$.

The orderings characterized in Propositions 2 and 3 are related, as shown in the following.

Note 3. Let $\mathbf{N}$ and $\mathbf{S} \in M_{n}^{>}$be fixed. For any $\mathbf{A}, \mathbf{B} \in M_{n}^{\geq}(\mathbf{S})$,

$\mathbf{A} \leq *_{\mathbf{N L}} \mathbf{B}$ if and only if $\mathbf{A} * \leq_{\mathbf{M L}} \mathbf{B}$ with $\mathbf{M}=\mathbf{S N}^{-1} \mathbf{S}$.

Pro of. This is a consequence of the equivalence of the following statements:

- $\mathrm{AN}^{-1} \mathrm{~A}^{*} \leq_{\mathrm{L}} \mathrm{BN}^{-1} \mathrm{~B}^{*}$

- $\mathrm{SAN}^{-1} \mathbf{A}^{*} \mathbf{S} \leq_{\mathrm{L}} \mathrm{SBN}^{-1} \mathbf{B}^{*} \mathrm{~S}$,

- $\mathbf{A}^{*} \mathbf{S N}^{-1} \mathbf{S A} \leq_{\mathrm{L}} \mathbf{B}^{*} \mathbf{S N}^{-1} \mathbf{S B}$,

- $\mathbf{A} * \leq_{\mathbf{M L}} \mathbf{B}$ with $\mathbf{M}=\mathbf{S N}^{-1} \mathbf{S}$.

Because of the above note, the Theorem below is only formulated for the right star NL-partial ordering.

Theorem. Let $\mathbf{S} \in M_{n}^{>}$be fixed. For any $\mathbf{A}, \mathbf{B} \in M_{n}^{\geq}(\mathbf{S})$,

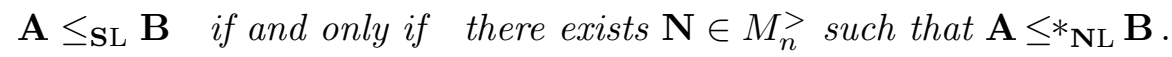

Proof. To prove the necessity observe that since $\mathbf{B}^{-} \mathbf{S}^{-1}=(\mathbf{S B})^{-}$and $R(\mathbf{A}) \subseteq R(\mathbf{B})$ by assumption, we have

$$
\mathbf{A B}^{-} \mathbf{A}=\mathbf{A B}^{-} \mathbf{S}^{-1} \mathbf{S A}=\mathbf{A}(\mathbf{S B})^{+} \mathbf{A}^{*} \mathbf{S} .
$$

Then, by Note $2, \mathbf{A} \leq_{\text {SL }} \mathbf{B}$ implies that $\mathbf{A}(\mathbf{S B})^{+} \mathbf{A}^{*} \leq_{\mathbf{L}} \mathbf{B S}^{-1}$, leading to $\mathbf{A} \leq *_{\mathrm{NL}} \mathbf{B}$ with $\mathbf{N}=\mathbf{Q}_{\mathbf{B}}+\mathbf{S B}$. 
The sufficiency follows from Proposition 1(d), Proposition 3(b) and from the following inequality which holds for all matrix norms:

$$
\varrho(\mathbf{C}) \leq\|\mathbf{C}\| ;
$$

cf. [7, p. 297].

For $\mathbf{S}=\mathbf{I}$, the result of the Theorem was proved independently in [3].

4. Results for the set of rectangular matrices. Propositions 1, 2 , and 3 , excluding parts $1(\mathrm{a}), 2(\mathrm{a})$, and $3(\mathrm{a})$, remain valid outside the cones $M_{n}^{\geq}(\mathbf{S})$-in the set $M_{n}$ or even in $M_{m, n}$. It is interesting to verify whether they still characterize an ordering or a preordering. In the case of Proposition 1, taking for example the nonsingular matrices

$$
\mathbf{A}=\left(\begin{array}{ll}
1 & 1 \\
0 & 1
\end{array}\right), \quad \mathbf{B}=\left(\begin{array}{ll}
1 & 0 \\
0 & 1
\end{array}\right), \quad \text { and } \quad \mathbf{C}=\left(\begin{array}{rr}
2 & -1 \\
-1 & 2
\end{array}\right)
$$

we have $\varrho\left(\mathbf{B}^{-1} \mathbf{A}\right)=1$ and $\varrho\left(\mathbf{C}^{-1} \mathbf{B}\right)=1$, but $\varrho\left(\mathbf{C}^{-1} \mathbf{A}\right)>1$, and hence we have neither an ordering nor a preordering. In the case of Proposition 2, taking for example $\mathbf{B}=-\mathbf{A}$, we see that the relation (b) is not antisymmetric, but its reflexivity and transitivity gives a preordering. The same conclusion is valid for Proposition 3. Remembering that the symbols in parts (a) of Propositions 2 and 3 define preorderings in the set $M_{m, n}$ we will compare these relations with star orderings.

The star ordering $\mathbf{A} \stackrel{*}{\leq} \mathbf{B}$, the left star ordering $\mathbf{A} * \leq \mathbf{B}$, and the right star ordering $\mathbf{A} \leq * \mathbf{B}$ are defined in $M_{m, n}$, respectively, by

$$
\begin{array}{rll}
\mathbf{A}^{*} \mathbf{A}=\mathbf{A}^{*} \mathbf{B} & \text { and } & \mathbf{A} \mathbf{A}^{*}=\mathbf{B} \mathbf{A}^{*}, \\
\mathbf{A}^{*} \mathbf{A}=\mathbf{A}^{*} \mathbf{B} & \text { and } & R(\mathbf{A}) \subseteq R(\mathbf{B}),
\end{array}
$$

and

$$
\mathbf{A A}^{*}=\mathbf{B A}^{*} \text { and } \quad R\left(\mathbf{A}^{*}\right) \subseteq R\left(\mathbf{B}^{*}\right) .
$$

The first ordering was introduced by Drazin [6], and Mitra [8] characterized it by

$$
\left\{\mathbf{B}_{l}^{-}\right\} \subseteq\left\{\mathbf{A}_{l}^{-}\right\} \quad \text { and } \quad\left\{\mathbf{B}_{m}^{-}\right\} \subseteq\left\{\mathbf{A}_{m}^{-}\right\} .
$$

The last two orderings were defined and characterized in [2], respectively, by

$$
\left\{\mathbf{B}_{l}^{-}\right\} \subseteq\left\{\mathbf{A}_{l}^{-}\right\}
$$

and

$$
\left\{\mathbf{B}_{m}^{-}\right\} \subseteq\left\{\mathbf{A}_{m}^{-}\right\}
$$

To compare these orderings with the preorderings defined in Propositions 2 and 3 in the set $M_{m, n}$ we need, in fact, more general forms of the 
latter, namely, the star $\mathbf{M N}$-ordering $\mathbf{A} \stackrel{*}{\leq}{ }_{\mathbf{M N}} \mathbf{B}$, the left star $\mathbf{M}$-ordering $\mathbf{A} * \leq_{\mathbf{M}} \mathbf{B}$, and the right star $\mathbf{N}$-ordering $\mathbf{A} \leq *_{\mathbf{N}} \mathbf{B}$ defined, respectively, by

$$
\begin{array}{lll}
\mathbf{A}^{*} \mathbf{M} \mathbf{A}=\mathbf{A}^{*} \mathbf{M B} & \text { and } & \mathbf{A} \mathbf{N}^{-1} \mathbf{A}^{*}=\mathbf{B N}^{-1} \mathbf{A}^{*}, \\
\mathbf{A}^{*} \mathbf{M} \mathbf{A}=\mathbf{A}^{*} \mathbf{M B} & \text { and } & R(\mathbf{A}) \subseteq R(\mathbf{B}),
\end{array}
$$

and

$$
\mathbf{A} \mathbf{N}^{-1} \mathbf{A}^{*}=\mathbf{B} \mathbf{N}^{-1} \mathbf{A}^{*} \text { and } \quad R\left(\mathbf{A}^{*}\right) \subseteq R\left(\mathbf{B}^{*}\right) .
$$

They can also be characterized, respectively, by

$$
\begin{gathered}
\left\{\mathbf{B}_{l(\mathbf{M})}^{-}\right\} \subseteq\left\{\mathbf{A}_{l(\mathbf{M})}^{-}\right\} \quad \text { and } \quad\left\{\mathbf{B}_{m(\mathbf{N})}^{-}\right\} \subseteq\left\{\mathbf{A}_{m(\mathbf{N})}^{-}\right\}, \\
\left\{\mathbf{B}_{l(\mathbf{M})}^{-}\right\} \subseteq\left\{\mathbf{A}_{l(\mathbf{M})}^{-}\right\},
\end{gathered}
$$

and

$$
\left\{\mathbf{B}_{m(\mathbf{N})}^{-}\right\} \subseteq\left\{\mathbf{A}_{m(\mathbf{N})}^{-}\right\} .
$$

We will characterize the above orderings in the way similar to the ones proposed in [8] and [2], and in Proposition 1.

Proposition 4. Let $\mathbf{M} \in M_{m}^{>}$and $\mathbf{N} \in M_{n}^{>}$be fixed. For any $\mathbf{A}, \mathbf{B} \in$ $M_{m, n}$, the following statements are equivalent:
(a) $\mathbf{A} * \leq_{\mathbf{M}} \mathbf{B}$,
(b) $\mathbf{A B}_{l(\mathbf{M})}^{-}=\mathbf{A} \mathbf{A}_{\mathbf{M N}}^{+}$for every $\mathbf{B}_{l(\mathbf{M})}^{-}$,
(c) $\mathbf{A} \mathbf{B}_{l(\mathbf{M}) r}^{-}=\mathbf{A} \mathbf{A}_{\mathbf{M N}}^{+}$for every $\mathbf{B}_{l(\mathbf{M}) r}^{-}$,
(d) $\mathbf{A} \mathbf{B}_{l(\mathbf{M})}^{-}=\mathbf{A} \mathbf{A}_{\mathbf{M} \mathbf{N}}^{+}$for some $\mathbf{B}_{l(\mathbf{M})}^{-}$and $R\left(\mathbf{A}^{*}\right) \subseteq R\left(\mathbf{B}^{*}\right)$,
(e) $\mathbf{A} \mathbf{B}_{\mathbf{M N}}^{+}=\mathbf{A} \mathbf{A}_{\mathbf{M N}}^{+}$and $R\left(\mathbf{A}^{*}\right) \subseteq R\left(\mathbf{B}^{*}\right)$,
(f) $\left\{\mathbf{B}_{l(\mathbf{M}) r}^{-}\right\} \subseteq\left\{\mathbf{A}_{l(\mathbf{M})}^{-}\right\}$.

Proposition 5. Let $\mathbf{M} \in M_{m}^{>}$and $\mathbf{N} \in M_{n}^{>}$be fixed. For any $\mathbf{A}, \mathbf{B} \in$ $M_{m, n}$, the following statements are equivalent:
(a) $\mathbf{A} \leq *_{\mathbf{N}} \mathbf{B}$,
(b) $\mathbf{B}_{m(\mathbf{N})}^{-} \mathbf{A}=\mathbf{A}_{\mathbf{M} \mathbf{N}}^{+} \mathbf{A}$ for every $\mathbf{B}_{m(\mathbf{N})}^{-}$,
(c) $\mathbf{B}_{m(\mathbf{N}) r}^{-} \mathbf{A}=\mathbf{A}_{\mathbf{M N}}^{+} \mathbf{A}$ for every $\mathbf{B}_{m(\mathbf{N}) r}^{-}$,
(d) $\mathbf{B}_{m(\mathbf{N})}^{-} \mathbf{A}=\mathbf{A}_{\mathbf{M N}}^{+} \mathbf{A}$ for some $\mathbf{B}_{m(\mathbf{N})}^{-}$and $R(\mathbf{A}) \subseteq R(\mathbf{B})$,
(e) $\mathbf{B}_{\mathbf{M} \mathbf{N}}^{+} \mathbf{A}=\mathbf{A}_{\mathbf{M} \mathbf{N}}^{+} \mathbf{A}$ and $R(\mathbf{A}) \subseteq R(\mathbf{B})$,
(f) $\left\{\mathbf{B}_{m(\mathbf{N}) r}^{-}\right\} \subseteq\left\{\mathbf{A}_{m(\mathbf{N})}^{-}\right\}$.

Conditions (f) in Propositions 4 and 5 follow from a result of [2], while the remaining conditions can be obtained from the results of [2] and [8].

The results of Propositions 2 and 3 and Propositions 4 and 5 are compared in the following.

Note 4. Let $\mathbf{M} \in M_{m}^{>}$and $\mathbf{N} \in M_{n}^{>}$be fixed. For any $\mathbf{A}, \mathbf{B} \in M_{m, n}$, the following implications hold: 
(a) $\mathbf{A} * \leq_{\mathbf{M}} \mathbf{B} \Rightarrow \mathbf{A} * \leq_{\mathbf{M L}} \mathbf{B}$,

(b) $\mathbf{A} \leq *_{\mathbf{N}} \mathbf{B} \Rightarrow \mathbf{A} \leq *_{\mathbf{N L}} \mathbf{B}$

(c) $\mathbf{A} \stackrel{*}{*}_{\mathbf{M N}} \mathbf{B} \Rightarrow \mathbf{A} * \leq_{\mathrm{ML}} \mathbf{B}$ and $\mathbf{A} \leq{ }^{*} \mathbf{N L}$ B.

It would be interesting to find a partial ordering $\leq *^{*}$ ? defined in $M_{m, n}$ for which

$$
\mathbf{A} \leq *_{\mathbf{N}} \mathbf{B} \Rightarrow \mathbf{A} \leq *_{?} \mathbf{B} \Rightarrow A \leq *_{\mathbf{N L}} \mathbf{B} .
$$

This is possible, for example, by modification of the left star M-partial ordering by using, instead of (4.1), the relations

$$
\mathbf{A}^{*} \mathbf{M A}=\lambda \mathbf{A}^{*} \mathbf{M B} \text { and } R(\mathbf{A}) \subseteq R(\mathbf{B}),
$$

with $\lambda \in[0,1]$. A similar extension can be done for the right star $\mathbf{N}$-partial ordering.

In the special case of the set $M_{m, 1}$, the ordering $\mathbf{a} \leq *_{\mathbf{N}} \mathbf{b}$ means that $\mathbf{a}=\mathbf{b}$ while its modification described above is equivalent to the relation $\mathbf{a}=\lambda \mathbf{b}$, where $\lambda \in[0,1]$.

In the general case a better modification might be possible.

\section{References}

[1] J. K. Baksalary, E. P. Liski and G. Trenkler, Mean square error matrix improvements and admissibility of linear estimators, J. Statist. Plann. Inference 23 (1989), 313-325.

[2] J. K. Baksalary and S. K. Mitra, Left-star and right-star partial orderings, Linear Algebra Appl. 149 (1991), 73-89.

[3] J. K. Baksalary, B. Schipp and G. Trenkler, Some further results on Hermitian-matrix inequalities, ibid. 160 (1992), 119-129.

[4] P. A. Bekker, The positive semidefiniteness of partitioned matrices, ibid. 111 (1988), 261-278.

[5] A. Ben-Israel and T. N. E. Greville, Generalized Inverses: Theory and Applications, Wiley, New York, 1974.

[6] M. P. Drazin, Natural structures on semigroups with involution, Bull. Amer. Math. Soc. 84 (1978), 139-141.

[7] R. A. Horn and C. R. Johnson, Matrix Analysis, Cambridge University Press, Cambridge, 1985.

[8] S. K. Mitra, The minus partial order and the shorted matrices, Linear Algebra Appl. 83 (1986), 1-27.

[9] C. R. Rao, Estimation of parameters in a linear model, Ann. Statist. 4 (1976), $1023-1036$.

[10] C. R. Rao and S. K. Mitra, Generalized Inverse of Matrices and Its Applications, Wiley, New York, 1971. 
[11] C. Stępniak, Two orderings on a convex cone of nonnegative definite matrices, Linear Algebra Appl. 94 (1987), 263-272.

JAN HAUKE AND AUGUSTYN MARKIEWICZ

DEPARTMENT OF MATHEMATICAL AND STATISTICAL METHODS

AGRICULTURAL UNIVERSITY OF POZNAŃ

UL. WOJSKA POLSKIEGO 28

60-637 POZNAŃ, POLAND

Received on 13.5.1992;

revised version on 6.9.1993 